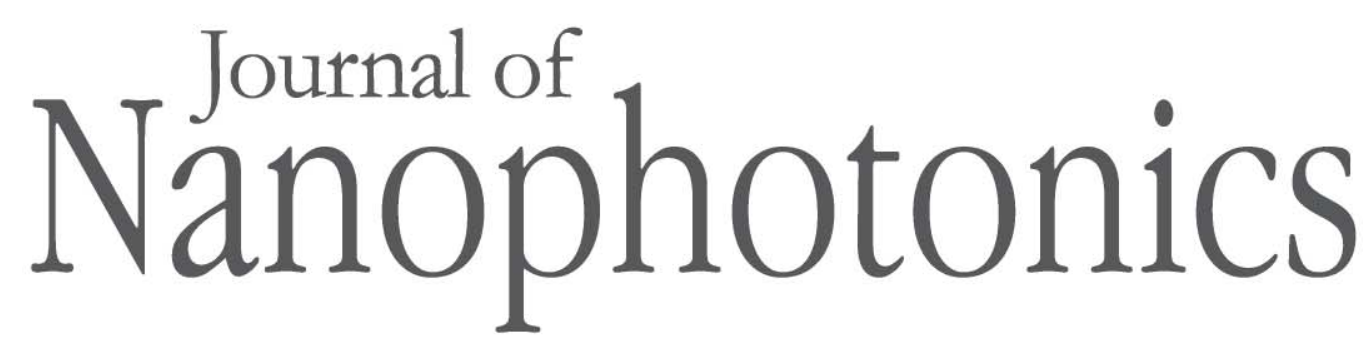

SPIEDigitalLibrary.org/jnp

\title{
Tutorial: Linear surface conductivity of an achiral single-wall carbon nanotube
}

Andrei M. Nemilentsau 


\title{
Tutorial: Linear surface conductivity of an achiral single-wall carbon nanotube
}

\author{
Andrei M. Nemilentsau \\ Belarus State University, Institute for Nuclear Problems, 11 Bobruiskaya Street, \\ Minsk 220030, Belarus \\ andrei.nemilentsau@gmail.com
}

\begin{abstract}
Theoretical consideration of electromagnetic scattering by single-wall carbon nanotubes (SWNTs) and SWNT arrays requires knowledge of the linear surface conductivity of an SWNT. An expression for the surface conductivity of an infinitely long SWNT was derived by Slepyan et al. [Phys. Rev. B 60, 17136-17149 (1999)]. The twin purposes of this tutorial are to succinctly discuss the derivation using the density matrix formalism and to provide ready-to-use expressions. @ 2011 Society of Photo-Optical Instrumentation Engineers (SPIE). [DOI: 10.1117/1.3574402]
\end{abstract}

Keywords: carbon nanotube; surface conductivity; density matrix.

Paper 11049L received Mar. 8, 2011; accepted for publication Mar. 14, 2011; published online Apr. 6, 2011.

\section{Introduction}

Peculiar electronic ${ }^{1}$ and optical properties ${ }^{2}$ of carbon nanotubes (CNTs) stimulate intensive studies of their electromagnetic response. Recently, the problem of the electromagnetic field diffraction by isolated CNTs, ${ }^{3-5}$ bundles, and composites ${ }^{6,7}$ containing CNTs received a lot of attention due to the potentiality of CNT-based materials for the effective manipulation of electromagnetic fields in the megahertz, gigahertz, and terahertz frequency ranges. The scattering of the electric near-fields by $\mathrm{CNTs}^{8,9}$ has important applications in the near-field optical microscopy and spectroscopy with the CNT-based probes. Theoretical consideration of these problems requires knowledge of the linear surface conductivity of CNTs. Though the expressions for the conductivity of a single-wall carbon nanotube (SWNT) were previously obtained in Ref. 10 I feel the necessity to present the results of Ref. 10 for the general audience. The main idea of the article is to explain the method of the SWNT surface conductivity calculation and to provide the reader with the ready-to-use expressions for the conductivity. For this purpose I use the density matrix formalism that was applied previously ${ }^{1-13}$ for studying the nonlinear optical properties of SWNTs. The outline of the paper is as follows: equations of motions for the density matrix are discussed in Sec. 2, and the expressions for the SWNT conductivity are presented in Sec. 3. The consideration is restricted to the case of achiral zigzag $(m, 0)$ SWNTs and armchair $(m, m)$ SWNTs.

\section{Equations of Motion for the Density Matrix of the $\pi$-Electron Subsystem in an SWNT}

Let us consider an infinitely long achiral SWNT of a cross-sectional radius $R_{c n}$ aligned parallel to the $z$-axis of the Cartesian coordinate system. Suppose that the SWNT is exposed to the plane monochromatic electromagnetic wave propagating normally to the SWNT axis and the electric field of the wave is polarized along the SWNT axis. As the typical SWNT radius (of order of $1 \mathrm{~nm}$ ) is small compared to the electromagnetic field wavelength up to the $\mathrm{x}$ ray frequency region we can neglect the space inhomogeneity of the electric field on the SWNT surface and

$1934-2608 / 2011 / \$ 25.00$ @ 2011 SPIE 
present it as follows:

$$
\mathbf{E}(t)=\mathbf{e}_{z} E(t)=\mathbf{e}_{z}\left(E_{0} e^{-i \omega t}+\text { c.c. }\right),
$$

where $\mathbf{e}_{z}$ is the unit vector along the $z$-axis, and $E_{0}$ and $\omega$ are the field amplitude and angular frequency, respectively.

The state of the $\pi$-electron subsystem in the SWNT can be characterized by the singleelectron density matrix $\rho_{\alpha \beta}\left(t, p_{z}, s\right)$, where $\alpha, \beta=c, v$, indices $c, v$ correspond to the conduction and valence bands, respectively, and $p_{z}$ is the projection of the electron quasimomentum on the SWNT axis. Due to the quantization of the electron quasimomentum component normal to the SWNT axis ${ }^{1}$ both the valence and the conduction bands of zigzag $(m, 0)$ and armchair $(m, m)$ SWNTs are split on $m$ sub-bands enumerated by an integer index $s=1, \ldots, m$. The equations of motion for the $\pi$-electron density matrix of the SWNT exposed to the electromagnetic field Eq. (1) has the following form ${ }^{11,12}$

$$
\begin{aligned}
\frac{\partial \rho\left(t, p_{z}, s\right)}{\partial t}+e E(t) \frac{\partial \rho\left(t, p_{z}, s\right)}{\partial p_{z}}= & -\frac{\rho\left(t, p_{z}, s\right)-\rho^{e q}\left(p_{z}, s\right)}{\tau_{1}} \\
& +\frac{2 i e}{\hbar} E(t) R_{c v}\left(p_{z}, s\right)\left[\rho_{c v}^{*}\left(t, p_{z}, s\right)-\rho_{c v}\left(t, p_{z}, s\right)\right], \\
\frac{\partial \rho_{c v}\left(t, p_{z}, s\right)}{\partial t}+e E(t) \frac{\partial \rho_{c v}\left(t, p_{z}, s\right)}{\partial p_{z}}= & -i \omega_{c v}\left(p_{z}, s\right) \rho_{c v}\left(t, p_{z}, s\right)-\frac{\rho_{c v}\left(t, p_{z}, s\right)}{\tau_{2}} \\
& -\frac{i e}{\hbar} E(t) R_{c v}\left(p_{z}, s\right) \rho\left(t, p_{z}, s\right),
\end{aligned}
$$

where $\rho=\rho_{c c}-\rho_{v v}$ is the dynamical inversion, $\rho^{e q}\left(p_{z}, s\right)$ is the equilibrium inversion, $\tau_{1,2}$ are the longitudinal and transversal electron relaxation times, $R_{c v}$ is the normalized matrix element of the electron dipole momentum operator, $\omega_{c v}$ is the frequency of the electron interband transitions, $e$ is the electron charge, $\hbar$ is the reduced Planck constant, and ${ }^{*}$ stands for complex conjugate.

Equations (2) and (3) take into account both intraband and direct interband transitions of $\pi$ electrons. Contribution of $\pi$ electrons indirect interband transitions to the electromagnetic response of an achiral SWNT can be neglected. As no assumption was made about the smallness of the electric field amplitude $E_{0}$ while deriving Eqs. (2) and (3), these equations can describe interaction between the SWNT and the electromagnetic field both in linear ${ }^{10}$ and non-linear regimes. ${ }^{11-13}$ As the single-electron approximation was used to obtain Eqs. (2) and (3), these equations can not account for the influence of the excitons on the electromagnetic properties of the SWNTs, though this influence can be essential in the optical frequency range. ${ }^{14}$

We are interested in the linear electromagnetic response of the SWNT. Using the perturbation theory we seek for the solution of Eqs. (2) and (3) in the form:

$$
\rho\left(t, p_{z}, s\right)=\rho^{e q}\left(p_{z}, s\right)+\lambda \rho^{(1)}\left(t, p_{z}, s\right), \quad \rho_{c v}\left(t, p_{z}, s\right)=\lambda \rho_{c v}^{(1)}\left(t, p_{z}, s\right),
$$

where $\lambda$ is an arbitrary small parameter, determining the strength of the perturbation. Substituting Eq. (4) in Eqs. (2) and (3), replacing $E(t)$ by $\lambda E(t)$ and collecting terms with the first power of $\lambda$ we obtain the system of uncoupled equations

$$
\begin{gathered}
\frac{\partial \rho^{(1)}\left(t, p_{z}, s\right)}{\partial t}+\frac{\rho^{(1)}\left(t, p_{z}, s\right)}{\tau_{1}}=-e E(t) \frac{\partial \rho^{e q}\left(p_{z}, s\right)}{\partial p_{z}}, \\
\frac{\partial \rho_{c v}^{(1)}\left(t, p_{z}, s\right)}{\partial t}+\left[i \omega_{c v}\left(p_{z}, s\right)+\frac{1}{\tau_{2}}\right] \rho_{c v}^{(1)}\left(t, p_{z}, s\right)=-\frac{i e}{\hbar} E(t) R_{c v}\left(p_{z}, s\right) \rho^{e q}\left(p_{z}, s\right),
\end{gathered}
$$


that can be easily solved by substitution

$$
\begin{gathered}
\rho^{(1)}\left(t, p_{z}, s\right)=\tilde{\rho}^{(1)}\left(\omega, p_{z}, s\right) E_{0} e^{-i \omega t}+\text { c.c. }, \\
\rho_{c v}^{(1)}\left(t, p_{z}, s\right)=\tilde{\rho}_{c v}^{(-)}\left(\omega, p_{z}, s\right) E_{0} e^{-i \omega t}+\tilde{\rho}_{c v}^{(+)}\left(\omega, p_{z}, s\right) E_{0} e^{i \omega t} .
\end{gathered}
$$

Taking into account the explicit form of incident field Eq. (1) and collecting terms with $e^{-i \omega t}$ we obtain:

$$
\begin{gathered}
\tilde{\rho}^{(1)}\left(\omega, p_{z}, s\right)=-\frac{i e}{\omega+i / \tau_{1}} \frac{\partial \rho^{e q}\left(p_{z}, s\right)}{\partial p_{z}}, \\
\tilde{\rho}_{c v}^{(\mp)}\left(\omega, p_{z}, s\right)=-\frac{e R_{c v}\left(p_{z}, s\right) \rho^{e q}\left(p_{z}, s\right)}{\hbar\left[\omega_{c v}\left(p_{z}, s\right) \mp \omega-i / \tau_{2}\right]} .
\end{gathered}
$$

\section{SWNT Conductivity}

Current density induced in an achiral SWNT by the electromagnetic field has the following form: ${ }^{11,12}$

$$
\begin{aligned}
j(t)= & \frac{2 e}{(2 \pi \hbar)^{2}} \frac{\hbar}{R_{c n}} \sum_{s=1}^{m} \int_{-a}^{a}\left\{\frac{\partial \mathcal{E}_{c}\left(p_{z}, s\right)}{\partial p_{z}} \rho^{(1)}\left(t, p_{z}, s\right)\right. \\
& \left.+i \omega_{c v}\left(p_{z}, s\right) R_{c v}\left(p_{z}, s\right)\left[\rho_{c v}^{(1) *}\left(t, p_{z}, s\right)-\rho_{c v}^{(1)}\left(t, p_{z}, s\right)\right]\right\} d p_{z},
\end{aligned}
$$

where $\mathcal{E}_{c}$ is the dispersion law of $\pi$ electrons in the SWNT, and $a$ defines the first Brillouin zone. Substituting expressions (7) and (8) into Eq. (11) we obtain

$$
j(t)=\sigma(\omega) E_{0} e^{-i \omega t}+\text { c.c. }
$$

where, by definition,

$$
\sigma(\omega)=\sigma_{1}(\omega)+\sigma_{2}(\omega)
$$

is the linear conductivity of the SWNT,

$$
\begin{gathered}
\sigma_{1}=-\frac{i e^{2}}{2 \pi^{2} R_{c n} \hbar\left(\omega+i / \tau_{1}\right)} \sum_{s=1}^{m} \int_{-a}^{a} \frac{\partial \mathcal{E}_{c}\left(p_{z}, s\right)}{\partial p_{z}} \frac{\partial \rho^{e q}\left(p_{z}, s\right)}{\partial p_{z}} d p_{z}, \\
\sigma_{2}=\frac{i e^{2}}{\pi^{2} R_{c n} \hbar^{2}} \sum_{s=1}^{m} \int_{-a}^{a} \frac{\omega_{c v}\left(p_{z}, s\right) R_{c v}^{2}\left(p_{z}, s\right) \rho^{e q}\left(p_{z}, s\right)\left(\omega+i / \tau_{2}\right)}{\omega_{c v}^{2}\left(p_{z}, s\right)-\left(\omega+i / \tau_{2}\right)^{2}} d p_{z} .
\end{gathered}
$$

Initially, Eqs. (13)-(15) were obtained in Ref. 10. Equations (14) and (15) account for the contribution of the intraband motions and interband transitions of the electrons to the total conductivity, respectively.

Define the functions that are present in Eqs. (14) and (15). The frequency of the electron interband transitions $\omega_{c v}$ and equilibrium inversion $\rho^{e q}$ are related to the dispersion law of $\pi$ 
electrons in the following way:

$$
\begin{gathered}
\omega_{c v}\left(p_{z}, s\right)=\frac{\mathcal{E}_{c}\left(p_{z}, s\right)-\mathcal{E}_{v}\left(p_{z}, s\right)}{\hbar}, \\
\rho^{e q}\left(p_{z}, s\right)=F\left[\mathcal{E}_{c}\left(p_{z}, s\right)\right]-F\left[\mathcal{E}_{v}\left(p_{z}, s\right)\right],
\end{gathered}
$$

where

$$
F(\mathcal{E})=\left[1+\exp \left(\frac{\mathcal{E}}{k_{B} T}\right)\right]^{-1}
$$

is the Fermi distribution, $k_{B}$ is the Boltzmann constant, and $T$ is the temperature. In order to proceed further, the SWNT type needs to be indicated. Using the tight-binding approximation it is easy to demonstrate $e^{1,10-12}$ that for zigzag $(m, 0)$ SWNT

$$
\begin{gathered}
\mathcal{E}_{c, v}\left(p_{z}, s\right)= \pm \gamma_{0}\left[1+4 \cos \left(\frac{3 b p_{z}}{2 \hbar}\right) \cos \left(\frac{\pi s}{m}\right)+4 \cos ^{2}\left(\frac{\pi s}{m}\right)\right]^{1 / 2}, \\
R_{c v}\left(p_{z}, s\right)=-\frac{b \gamma_{0}^{2}}{2 \mathcal{E}_{c}^{2}\left(p_{z}, s\right)}\left[1+\cos \left(\frac{3 b p_{z}}{2 \hbar}\right) \cos \left(\frac{\pi s}{m}\right)-2 \cos ^{2}\left(\frac{\pi s}{m}\right)\right], \\
R_{c n}=\frac{\sqrt{3} b m}{2 \pi}, \quad a=\frac{2 \pi \hbar}{3 b},
\end{gathered}
$$

while for armchair $(m, m)$ SWNT

$$
\begin{gathered}
\mathcal{E}_{c, v}\left(p_{z}, s\right)= \pm \gamma_{0}\left[1+4 \cos \left(\frac{\sqrt{3} b p_{z}}{2 \hbar}\right) \cos \left(\frac{\pi s}{m}\right)+4 \cos ^{2}\left(\frac{\sqrt{3} b p_{z}}{2 \hbar}\right)\right]^{1 / 2}, \\
R_{c v}\left(p_{z}, s\right)=-\frac{\sqrt{3} b \gamma_{0}^{2}}{2 \mathcal{E}_{c}^{2}\left(p_{z}, s\right)} \sin \left(\frac{\sqrt{3} b p_{z}}{2 \hbar}\right) \sin \left(\frac{\pi s}{m}\right), \\
R_{c n}=\frac{3 b m}{2 \pi}, \quad a=\frac{2 \pi \hbar}{\sqrt{3} b},
\end{gathered}
$$

where $\gamma_{0} \approx 2.7 \mathrm{eV}$ (Ref. 1) is the transfer integral, $b=0.142 \mathrm{~nm}$ is the interatomic distance in the SWNT.

Finally, let us obtain a simplified expression for the low-frequency conductivity of metallic SWNTs. Before doing so, recall that zigzag $(m, 0)$ SWNTs are metals when index $m$ is a multiple of 3 , while armchair $(m, m)$ SWNTs are always metals. When the frequency of the external electromagnetic field is below the frequency of the interband electron transitions, the intraband motions of the electrons give a dominant contribution to the conductivity of metallic SWNTs. This means that the low-frequency conductivity of a metallic SWNT is defined by Eq. (14) with great accuracy. Equation (14) can be further simplified for SWNTs of not very big radii $(m<50)$ as the main contribution to the integral is due to sub-bands $s$ crossing the Fermi level. The Fermi surface in SWNTs is defined by the set of points satisfying the condition $\mathcal{E}_{c, v}\left(p_{F}, s\right)=0$. In the vicinity of the Fermi level the dispersion law of electrons in metallic SWNTs has the following approximated form: ${ }^{1}$ 
Nemilentsau: Tutorial: Linear surface conductivity of an achiral single-wall...

$$
\mathcal{E}_{c, v}\left(p_{z}, s\right) \approx \pm v_{F}\left|p_{z}-p_{F}\right|
$$

where $v_{F}=3 b \gamma_{0} / 2 \hbar$ is the Fermi velocity. The contribution of the vicinity of each Fermi point $p_{F}$ to the integral in Eq. (14) is equal to

$$
\begin{aligned}
\int_{p_{F}-\Delta p_{F}}^{p_{F}+\Delta p_{F}} \frac{\partial \mathcal{E}_{c}}{\partial p_{z}} \frac{\partial \rho^{e q}}{\partial p_{z}} d p_{z} & =2 \int_{p_{F}}^{p_{F}+\Delta p_{F}}\left(\frac{\partial \mathcal{E}_{c}}{\partial p_{z}}\right)^{2} \frac{\partial \rho^{e q}}{\partial \mathcal{E}_{c}} d p_{z} \\
& \approx 2 v_{F} \int_{0}^{\mathcal{E}_{c}\left(p_{F}+\Delta p_{F}, s\right)} \frac{\partial \rho^{e q}}{\partial \mathcal{E}_{c}} d \mathcal{E}_{c} \\
& =2 v_{F}\left\{\rho^{e q}\left[\mathcal{E}_{c}\left(p_{F}+\Delta p_{F}, s\right)\right]-\rho^{e q}(0)\right\} \\
& =-2 v_{F},
\end{aligned}
$$

where we take into the account that $\rho^{e q}(0)=0$ and $\rho^{e q}\left(\mathcal{E}_{c}\right) \rightarrow-1$ for $\mathcal{E}_{c} \gg k_{B} T$. The last condition can be reformulated as $\Delta p_{F} / a \gg k_{B} T / \pi \gamma_{0}$ for zigzag SWNTs and $\Delta p_{F} / a \gg k_{B} T / \sqrt{3} \pi \gamma_{0}$ for armchair ones. As at the room temperature $k_{B} T \approx 0.025 \mathrm{eV}$ and $\gamma_{0} \approx 2.7 \mathrm{eV}$ these conditions are fulfilled for the nearest proximity of the Fermi point.

In zigzag SWNT sub-bands $s=m / 3$ and $s=2 m / 3$ crosses the Fermi level ones. In armchair SWNTs the sub-band $s=m$ crosses the Fermi level twice. This means that the integral in Eq. (14) is approximately equal to $-4 v_{F}$ both for metallic zigzag and armchair SWNTs. Thus, the following approximated expression for the conductivity of metallic SWNTs can be obtained:

$$
\sigma=i \frac{3 b \gamma_{0} e^{2}}{\pi^{2} R_{c n} \hbar^{2}\left(\omega+i \tau_{1}\right)}
$$
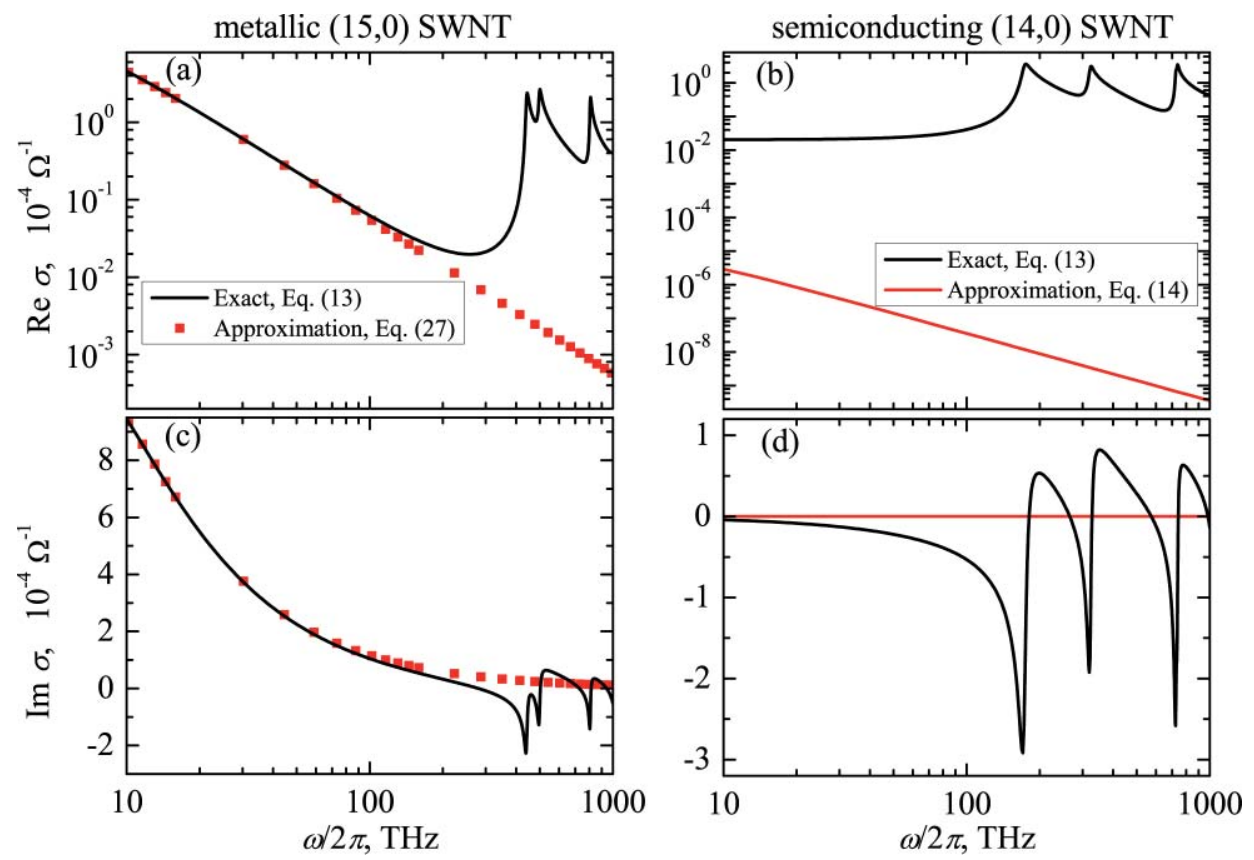

Fig. 1 Linear surface conductivity of (a) and (c) metallic $(15,0)$ SWNT and (b) and (d) semiconducting $(14,0)$ SWNT calculated using exact Eq. (13) and approximated Eqs. (14) and (27). $\tau_{1}=\tau_{2}=33$ fs in accordance with Ref. 6 . 
where the SWNT radius is defined by Eq. (21) for zigzag SWNTs and Eq. (24) for armchair ones.

Conductivity spectra of semiconducting $(14,0)$ SWNT and metallic $(15,0)$ SWNT are presented in Fig. 1. As one can see, in the low-frequency region the values of the conductivity of $(15,0)$ SWNT calculated using approximated Eq. (27) are in good agreement with the conductivity values obtained by Eq. (13). However, in the high-frequency region contribution of the interband electron transitions becomes dominant and approximation (27) is no more applicable. In contrast to metallic SWNTs, the conductivity band of semiconducting SWNTs is practically unoccupied at room temperature. This means that the contribution of the intraband electron motions to the conductivity of semiconducting SWNTs is always small and thus the contribution of the interband electron transitions given by Eq. (15) always needs to be accounted for. Note that surface conductivity unit $\Omega^{-1}$ differs from the volume conductivity unit $\Omega^{-1} \mathrm{~cm}^{-1}$.

\section{Concluding Remarks}

In this tutorial the method of the SWNT surface conductivity calculation is discussed. Equations of motions for the density matrix of the $\pi$-electron subsystem in the SWNT illuminated by the plane monochromatic electromagnetic wave were solved analytically by the perturbation method and the expressions for the linear conductivity of achiral SWNTs were obtained. All the parameters and functions necessary to calculate the SWNT conductivity without referring to any other sources are indicated. Both SI and Gaussian units can be used.

\section{Acknowledgments}

This research was partially supported by the Belarus Republican Foundation for Fundamental Research under Project Nos. F10CO-020 and N. EU FP7 under Project No. FP7266529 BY-NanoERA. A.M. is grateful to Dr. Sergey Maksimenko, Dr. Gregory Slepyan, and Professor Akhlesh Lakhtakia for their invaluable help in developing the methods presented in the tutorial.

\section{References}

1. J.-C. Charlier, X. Blase, and S. Roche, "Electronic and transport properties of nanotubes," Rev. Mod. Phys. 79, 677-732 (2007).

2. Ph. Avouris, M. Freitag, and V. Perebeinos, "Carbon-nanotube photonics and optoelectronics," Nat. Photon. 2, 341-350 (2008).

3. G. Hanson, "Fundamental transmitting properties of carbon nanotube antennas," IEEE Trans. Antennas Prop. 53, 3426-3435 (2005).

4. G. Ya. Slepyan, M. V. Shuba, S. A. Maksimenko, and A. Lakhtakia, "Theory of optical scattering by achiral carbon nanotubes and their potential as optical nanoantennas," Phys. Rev. B 73, 195416 (2006).

5. C. Rutherglen and P. Burke, "Nanoelectromagnetics: Circuit and electromagnetic properties of carbon nanotubes," Small 5(8), 884-906 (2009).

6. G. Ya. Slepyan, M. V. Shuba, S. A. Maksimenko, C. Thomsen, and A. Lakhtakia, "Terahertz conductivity peak in composite materials containing carbon nanotubes: Theory and interpretation of experiment," Phys. Rev. B 81, 205423 (2010).

7. A. M. Nemilentsau, M. V. Shuba, G. Ya. Slepyan, P. P. Kuzhir, S. A. Maksimenko, P. N. D'yachkov, and A. Lakhtakia, "Substitutional doping of carbon nanotubes to control their electromagnetic characteristics," Phys. Rev. B 82, 235424 (2010).

8. A. M. Nemilentsau, G. Y. Slepyan, S. A. Maksimenko, A. Lakhtakia, and S. V. Rotkin, "Scattering of the near field of an electric dipole by a single-wall carbon nanotube," $J$. Nanophoton. 4, 041685 (2010). 
9. A. M. Nemilentsau, G. Ya. Slepyan, S. A. Maksimenko, A. Lakhtakia, and S. V. Rotkin, "Spontaneous decay of the excited state of an emitter near a finite-length metallic carbon nanotube," Phys. Rev. B 82, 235411 (2010).

10. G. Ya. Slepyan, S. A. Maksimenko, A. Lakhtakia, O. Yevtushenko, and A. V. Gusakov, "Electrodynamics of carbon nanotubes: Dynamic conductivity, impedance boundary conditions, and surface wave propagation," Phys. Rev. B 60, 17136-17149 (1999).

11. G. Ya. Slepyan, A. A. Khrutchinskii, A. M. Nemilentsau, S. A. Maksimenko, and J. Herrmann, "High-order optical harmonic generation on carbon nanotubes: Quantummechanical approach," Int. J. Nanosci. 3(3), 343-354 (2004).

12. A. M. Nemilentsau, G. Ya. Slepyan, A. A. Khrutchinskii, and S. A. Maksimenko, "Thirdorder optical nonlinearity in single-wall carbon nanotubes," Carbon 44(11), 2246-2253 (2006).

13. A. M. Nemilentsau, G. Ya. Slepyan, and S. A. Maksimenko, "Rabi oscillations and saturable absorption effect in single-wall carbon nanotubes," J. Phys. Conf. Ser. 248(1), 012015 (2010).

14. J. Maultzsch, R. Pomraenke, S. Reich, E. Chang, D. Prezzi, A. Ruini, E. Molinari, M. S. Strano, C. Thomsen, and C. Lienau, "Exciton binding energies in carbon nanotubes from two-photon photoluminescence," Phys. Rev. B 72, 241402 (2005).

Biography and photograph of the author not available. 